International Journal of Stem Cell Research and Transplantation (1JST)

ISSN 2328-3548

\title{
Gene Expression Analysis of Rat Adipose Tissue-Derived Stem Cells
}

\author{
Research Article \\ Cannella V ${ }^{1}$, Casella $\mathrm{S}^{2}$, Altomare $\mathrm{R}^{3}$, Lo Monte $\mathrm{AI}^{3}$, Di Marco $\mathrm{P}^{1}$, Russotto L ${ }^{1}$, Di Bella $\mathrm{S}^{1}$, Purpari $\mathrm{G}^{1}$, Gucciardi F $\mathrm{F}^{1}$, Cassata $\mathrm{G}^{1}$, Piccione $\mathrm{G}^{2}$, Guer- \\ cio $\mathrm{A}^{1}$
}

${ }^{1}$ Area Diagnostica Virologica dell’Istituto Zooprofilattico Sperimentale della Sicilia “A. Mirri”. Palermo, Italy.

${ }^{2}$ Dipartimento di Scienze Veterinarie, Università degli Studi di Messina, Messina. Italy.

${ }^{3}$ Dipartimento CHIRON, Complesso Chirurgia generale e Trapianti, Università degli Studi di Palermo, Palermo, Italy.

\begin{abstract}
The aim of this study was to isolate and characterize rat Adipose Derived Mesenchymal Stem Cells (AD-MSCs) in order to evaluate the stemness markers gene expression levels, together with the assessment of phenotypic expression of the CD90 and CD45 markers. MSCs were obtained from subcutaneous adipose tissue of $16 \mathrm{Wistar}$ rats. Microbiological controls were performed to exclude the presence of bacteria, fungi and viruses in tissue. Adipose tissue was mechanically and enzimatically fragmented and stromal cell fraction was seeded in adherent culture flasks in DMEM $20 \%$ FBS. After 48h, the medium was replaced. Cells were characterized by evaluating their ability to adhere to the plastic; the clonogenic potential by Colony Forming Unit (CFU) assay and their ability to differentiate adipocytes and osteocytes. AD-MSCs were analysed for the expression of three stem cell master genes (OCT-4, SOX-2 and NANOG) at different cell subcultures by Sybr Green RealTime PCR. Statistical analysis, performed with REST software, showed that the expression of the genes is maintained from subculture 0 to 5 . Moreover, flow cytometry analysis confirmed the mesenchymal nature of cells isolated from adipose tissue as they were positive for CD90, which is a MSCs surface marker, and negative for CD45 (typical hematopoietic marker).
\end{abstract}

Keywords: Adipose-Derived Mesenchymal Stem Cells; Gene Expression; Immunophenotypical Analysis; Rat.

\section{*Corresponding Author:}

Dr. Stefania Casella,

Dipartimento di Scienze Veterinarie, Università degli Studi di Messina,

Polo Universitario dell'Annunziata, 98168 Messina, Italy.

Tel: +390903503520

Fax: +39090 3503975

Email: stefania.casella@unime.it

Received: July 02, 2015

Accepted: November 23, 2015

Published: November 24, 2015

Citation: Casella S, et al., (2015) Gene Expression Analysis of Rat Adipose Tissue-Derived Stem Cells. Int J Stem Cell Res Transplant 03(5), 120124. doi: http://dx.doi.org/10.19070/2328-3548-1500020

Copyright: Casella S S $^{\odot}$ 2015. This is an open-access article distributed under the terms of the Creative Commons Attribution License, which permits unrestricted use, distribution and reproduction in any medium, provided the original author and source are credited.

\section{Introduction}

Over the past few years in veterinary medicine there has been an increased interest in understanding the biology of stem cells. They are undifferentiated cells from the embryo, fetus or adult that have the unique potential to generate various differentiated tissue cells under appropriate biochemical, hormonal and mechanical stimuli in vitro and in vivo [1]. Particularly, adult stem cells represent a promising therapeutic tool especially in wound repair, tissue engineering and application in therapeutic fields $[2,3]$. These cells can be isolated from a variety of tissues and organs including peripheral blood, central nervous system, muscle, umbilical cord, bone marrow and adipose tissue [4-8]. Mesenchymal stem cells (MSCs) derived from bone marrow (BM-MSCs) and adipose tissue (AD-MSCs) are the most highly characterized and are quite comparable [9]. Both have broad multipotency with differentiation into different cell lineages, including adipo-, osteo- and chondrocytic lineages $[9,10]$. Their stromal vascular fraction contains stem cells, T-lymphocytes, anti-inflammatory macrophages, endothelial precursor cells, and preadipocytes; they can differentiate into bone, cartilage, muscle and neuronal cells [11]. Previous studies conducted on adult stem cell therapy have focused on BM-MSCs, however the easy and repeatable access to adipose tissue, the simpler isolation procedure and the greater numbers of fresh MSCs derived from equivalent amounts of fat versus bone marrow provide a clear advantage in using AD-MSCs compared to BM-MSCs [11-13]. AD-MSCs, in fact, can be easily cultured and propagated more rapidly, retaining their mesenchymal multipotency after some passages [14]. For these reasons, AD-MSCs have been isolated and characterized by several researchers in human medicine [15] and in a variety of animals [16]. Some authors have referred the isolation and characterization of equine amnion mesenchymal stem cells [17] whereas in dog and in mouse the isolation and characterization of MSCs have been reported from subcutaneous adipose tissue $[2,18-20]$. Characterization of MSCs is based mainly on the expression of some markers as CD105, CD73 and CD90 and on the absence of expression of some hematopoietic markers CD34, CD45, CD14 o CD11b, CD19 e CD79a. Moreover, it is known that the pluripotency of a stem cell depends on the expression of genes that code for transcription factors such as OCT4, NANOG and SOX-2. In rat, some researchers have isolated and characterized bone marrow stromal cells [21]; however, there are a few reports on rat AD-MSCs [22]. 
Considering that the rat could be a good model for the research purpose and that some variations occur among species (although MSCs from different species have similar characteristics) and that the gene expression and the phenotypic characterization of ADMSC is still controversial, the aim of this study was to describe the isolation and characterization of rat AD-MSCs evaluating the stemness marker gene expression levels together with the assessment of phenotypic expression of the CD90 and CD45 markers.

\section{Experimental Procedures}

\section{AD-MSC isolation, characterization and quality controls}

Adipose tissue samples, obtained from 16 Wistar rats, ranging in age from 1 to 3 years and a mean body weight of $350 \pm 30 \mathrm{~g}$ were used for the study. All protocols used in the present study were reviewed and approved in accordance with the standards recommended by the Guide for the Care and Use of Laboratory Animals and by Directive 2010/63/UE. AD-MSCs were obtained collecting subcutaneous fat depots from each rat, using standard surgical procedures and a mild sedation by injection of Medetomidine $(0.5 \mathrm{ml} / \mathrm{kg}$ body weight) in according to DL 116/92 and Directive 2010/63/UE. AD-MSCs isolated, were amplified in vitro and successively characterized.

Isolation was performed by digestion of adipose tissue for 3-4 hours at $37^{\circ} \mathrm{C}$ with collagenase type IA $0.2 \%$ (Sigma, St. Louis), prepared in sterile phosphate-buffered saline (PBS) solution, containing $1 \%$ antibiotics (penicillin, streptomycin and amphotericin) as described previously by Guercio et al. 2012. After 48 h, unattached cells were removed by washing with Hank's Balanced Salt Solution (HBSS, Gibco ${ }^{\circledR}$ ), supplemented with $10 \%$ antibiotics (penicillin, streptomycin and amphotericin) and the culture medium was renewed. Adherent cells, grown to semiconfluence, were harvested, quantified and subcultured. To perform subsequent AD-MSCs subcultures, a solution made with $0.05 \%$ of Trypsin and $0.02 \%$ of EDTA was used. Cells were also cryopreserved, for further studies, in D-MEM Low Glucose medium with $20 \%$ FBS and 10\% dimethyl sulfoxide (DMSO, Sigma-Aldrich ${ }^{\circledR}$ ). The identity of AD-MSCs was verified by assessing either their ability to attach to the plastic surface of culture flasks, to form colonyforming units (CFU assay) and to differentiate in cells derived from mesodermal lineages, adipocytes and osteocytes, in presence of appropriated culture media. In order to induce adipogenic differentiation, cells were plated in six-well plates, and treated with the Complete MesenCult ${ }^{\circledR}$ Adipogenic Medium (Stemcell Technologies) for 21 days, with medium change once in every 3 days. Oil Red $\mathrm{O}$ staining was done to examine the lipid droplet for- mation. Osteogenesis was instead inducted by plating the cells in six-well plates with the NH OsteoDiff Medium (Mylteny Biotec) for 3 weeks, with medium change once in every 3 days. Von Kossa staining was carried out to detect calcified extracellular matrix deposits. Tumorigenic control of AD-MSCs was assessed in vitro [2]. The AD-MSCs, the cell line VERO (negative control) and the cell line Hep-2 (positive control) were cultured in vitro into three different flasks. Later, each cell culture was inoculated in 6-well plates, which contained the solid medium, and incubated at $37^{\circ} \mathrm{C}$. They were observed by microscopy for 3 weeks. After 7-10 days, the tumorigenic cells started to replicate, producing multicellular agglomerates. The negative cells showed atrophy. Moreover, adipose tissue collected and AD-MSCs were tested for a putative microbiological contamination during the steps of production. For this purpose, an aliquot of fat and of supernatant of cell culture was inoculated into specifics bacteriological culture media. Mycoplasma detection was performed through a specific Real Time PCR, while the potential Pestiviruses contamination was detected through a specific Real Time RT-PCR [2]. The presence of transcription factors indicative of self-renewal and undifferentiation was also investigated, together with the phenotypic assessment of membrane CD markers.

\section{Characterization of AD-MSCs: gene expression analysis}

Total RNAs were extracted from cells cultivated from subculture 0 to 5, using High Pure RNA isolation kit (Roche), according to the manufacturer's instructions. cDNAs were synthesized from total RNA using random hexamers and Superscript Vilo cDNA Synthesis kit (Invitrogen).

Specific primers were designed using Primer Express software, to amplify fragments of Rattus_norvegicus stemness genes (OCT4: NM_001009178.2; NANOG: NM_001100781.1; SOX2: NM_001109181.1). Gene expression levels of selected genes were tested together with GADPH gene (XR598347.1), used as reference (Table 1).

All reactions (in triplicate) were performed in a final volume of $20 \mu \mathrm{L}$, containing $2 \mu \mathrm{L}$ of cDNA, 1X Sybr Green Buffer (Fast Sybr Green master mix - Applied Biosystems) and $1 \mu \mathrm{M}$ of each primer. Cycling conditions were as follows: $95^{\circ} \mathrm{C}$ for $10 \mathrm{~min} ; 40$ cycles at $95^{\circ} \mathrm{C}$ for $30 \mathrm{sec}, 60^{\circ} \mathrm{C}$ for $1 \mathrm{~min}$. Melting curve cycles were set as follows: $95^{\circ} \mathrm{C}$ for $15 \mathrm{sec}, 60^{\circ} \mathrm{C}$ for $1 \mathrm{~min}$ and $95^{\circ} \mathrm{C}$ for $15 \mathrm{sec}$. The dissociation curve was used to confirm the specificity of the amplicon. The relative levels of each RNA were calculated by the $2-\Delta \Delta$ CT method (CT represents the cycle number at which the signal reaches the threshold of detection) [23]. Each CT value,

Table 1. Primers used for gene expression.

\begin{tabular}{|c|c|}
\hline PRIMERS & GENE SEQUENCE \\
\hline GADPH-F & 5'-AAC 'T'T GGC ATC GTG GAA GGG-3' \\
\hline GADPH-R & 5'-AGG GAT GAT GT'T CTG GGC TGC-3' \\
\hline OCT4-F & 5'-GAG GCC TT'T CCC TCT GT'T CCT-3' \\
\hline OCT4-R & 5'-GGC TGG TGC CTC AGT T'TG AA-3' \\
\hline SOX-2-F & 5'-ACT GT'T T'T'T TCA TCC CAA T'TG CA-3' \\
\hline SOX-2-R & 5'-CGG AGA TCT GGC GGA GAA TA-3' \\
\hline NANOG-F & 5'-TCA GCG CCG GTG GAG TA-3' \\
\hline NANOG-R & 5'-TCC AGA CGC GT'T CAT CAG ATA G-3' \\
\hline
\end{tabular}


used for these calculations, is the mean of three replicates of the same reaction. To assess variations in gene expression during different subcultures, REST (Relative Expression Software Tool) 2009 software, V.2.0.13. was used. Only P-values $\leq 0.05$ were considered statistically significant. The statistical analysis performed through the use of REST 2009 was conducted by comparing the expression levels of stemness genes OCT-4, SOX-2 and NANOG in AD-MSC from subculture 1 to 5 (sample group) to the expression levels of the same genes at subculture 0 (control group).

\section{Characterization of AD-MSCs: cell immunophenotypical analysis}

Immunophenotypical analysis was performed on AD-MSCs cultivated in D-MEM from subculture 0 to 10 . Flow cytometric analysis was carried out through the use of a FACS CANTO II platform, with six channels (Becton Dickinson). For this purpose, two tubes, each containing $200 \mu \mathrm{L}$ of cell suspension, were set up: one without antibodies and the other with cells stained with antibodies against CD90 and CD45. Particularly, the cells harvested were centrifuged for $10 \mathrm{~min}$ at $400 \mathrm{~g}$ and stained at room temperature for $30 \mathrm{~min}$ with allophycocyanin (APC) conjugated mouse anti rat CD90 and fluorescein isothiocyanate (FITC) conjugated mouse anti rat CD 45 , at the concentration of $0.2 \mathrm{mg} / \mathrm{ml}$. First of all, the tube with cell sample without antibodies was acquired as negative control and used to set up the gating on the cell population of interest. For each tube 10.000 events were acquired.

\section{Results}

AD-MSCs were successfully isolated from rat adipose tissue. They became semi-confluent ( $80 \%$ ) in $25 \mathrm{~cm}^{2}$ flasks in 5-6 days, showing a fibroblast-like cells phenotype until 10 subculture (Figure 1). The CFU assay performed on AD-MSCs at the cell passages 1, 3 and 5 , showed the clonogenic efficiency of cells in culture that formed colonies on a plastic surface in D-MEM Low Glucose with $5 \%$ FBS.

AD-MSCs showed the ability to differentiate into two different mesodermal lineages tested, when cultivated with appropriated culture medium. After 4-5 days, AD-MSCs cultured in MesenCult $^{\circledR}$ Adipogenic Medium, formed lipid globules into the cytoplasm, stained positive with Oil Red O, which continued to increase in size. No differentiation was observed in the untreated cells.

AD-MSCs cultured in NH OsteoDiff Medium showed a cuboidlike phenotype and formed cell aggregates. Deposition of calcified extracellular matrix was highlighted after von Kosssa staining in treated cells, whereas the untreated cells showed unchanged phenotype.
Microbiological quality controls performed on adipose tissue and on stem cells in culture resulted negative for bacteria, fungi, Mycoplasma and Pestiviruses. This showed that manipulation in vitro was conducted under appropriate conditions of sterility. Tumorigenicity assay was negative.

\section{Gene expression analysis}

The expression levels of stemness genes in AD-MSCs quantified from subculture 0 to 5 are expressed as the average Delta $\mathrm{Ct}(\Delta \mathrm{Ct})$ \pm standard deviation (SD). $\Delta \mathrm{Ct}$ derived from the difference between the CT of each gene and the CT of the housekeeping gene GAPDH. For the calculation of the averages only samples with CT value lower than 40 were considered (Figure 2).

The statistical analysis showed no statistically significant differences on the expression of the three stemness genes since their expression levels were almost kept constant up to the fifth passage.

\section{Characterization of AD-MSCs: cell immunophenotypical analysis.}

Flow cytometric analysis, performed on AD-MSCs cultivated in D-MEM medium from subculture 0 to 10 , showed the presence of surface antigen CD90 and the simultaneous absence of CD45. Particularly, Figure 3 shows that $93.7 \%$ of selected cell population expressed CD90 at subculture 0 while $98.1 \%$ of the selected cell population expressed CD90 at subculture 10. This result confirmed the mesenchymal nature of AD-MSCs isolated.

\section{Discussion}

In the present study, we report the isolation and characterization in vitro of MSCs from the subcutaneous fat of rat. By the use of different techniques, we characterized the AD-MSCs isolated, providing experimental evidences of their mesenchimal nature. The obtained results agree with the findings obtained in an our previous study utilizing the same techniques [22].

A limited number of studies have been performed to assess the expression of stemness markers, showing controversial findings respect to our results. Disagree with some authors [24] that demonstrated that mouse adipose tissue-derived stem cells do not express the OCT-4 markers and agree with Izadpanah et al. [25] that showed that OCT4 and SOX2 are abundantly expressed in cultured rhesus and human AD-MSC, our results showed the presence of a significative expression of stemness marker genes, OCT4, SOX2 and NANOG. The study of gene expression levels, was performed on the basis of related mRNA levels, evaluated during five subsequent subcultures. The analysis used, highlighted almost constant levels of gene expression for the three stemness

Figure 1. Adipose tissue-derived stem cells of rat at different subcultures (0, 6 and 10).

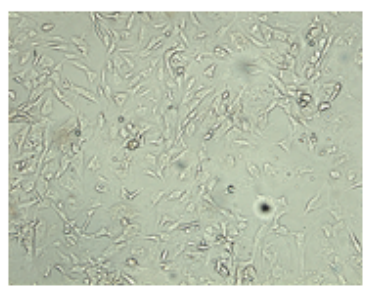

Subculture 0

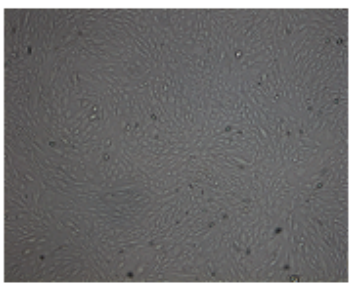

Subculture 6

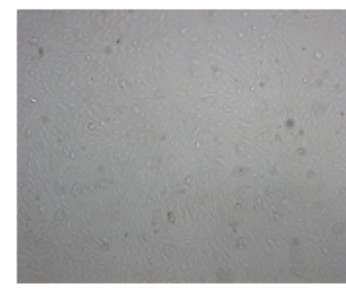

Subculture 10 
Figure 2. Levels of stemness genes expressed in AD-MSCs (OCT4, SOX-2 and NANOG) quantified from subculture 0 to 5. The values are expressed as average Delta $\mathrm{Ct}(\Delta \mathrm{Ct}) \pm$ standard deviation (SD).

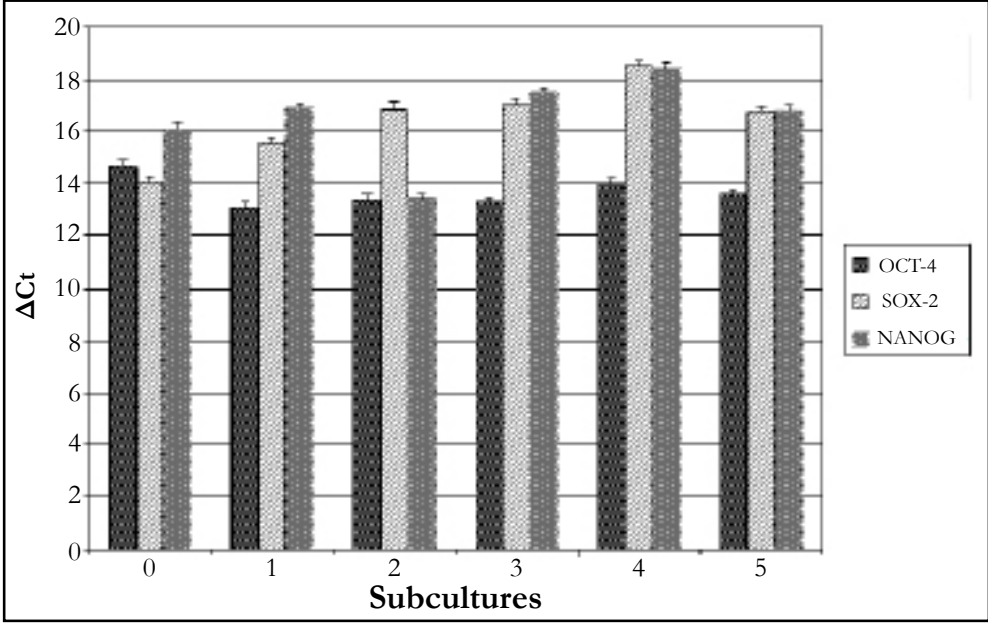

Figure 3. Immunophenotypic analysis of AD-MSCs for CD90 and CD45 expression, from subculture 0 (on the top) to subculture 10 (on the bottom).
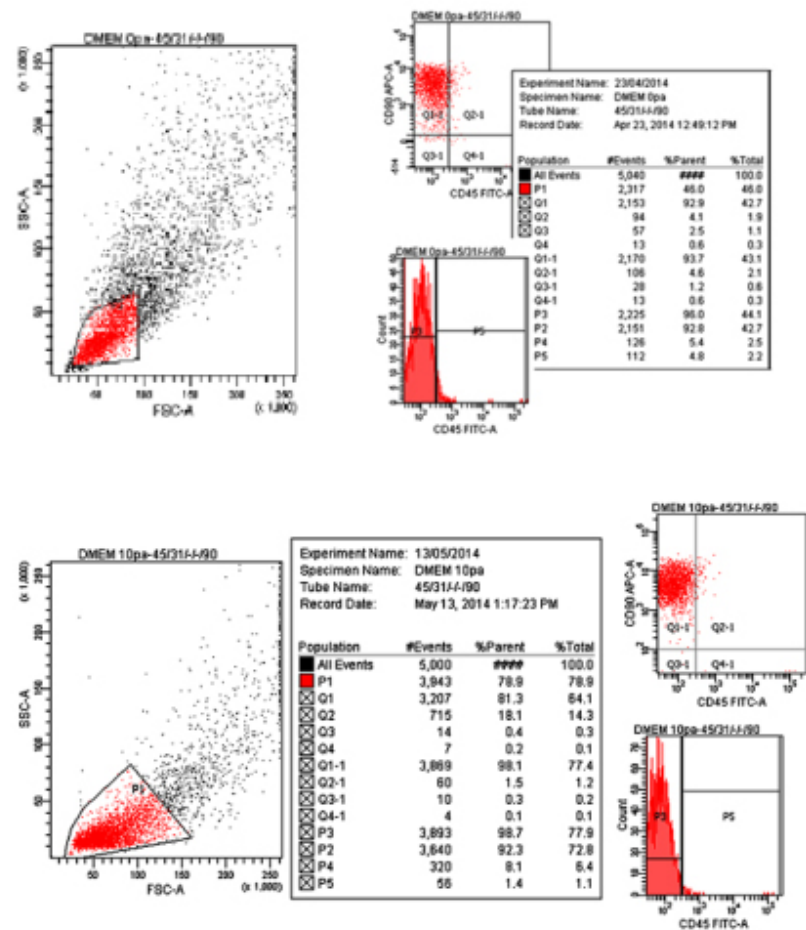

markers, during the different subcultures. This showed that isolated cells maintain their mesenchymal nature. These data were also confirmed by flow cytometry analysis that showed the presence of CD90 surface marker and the absence of CD45 up to 10 subcultures, during which AD-MSCs maintain the typical fibroblastoid morphology. In addition, isolated AD-MSCs also proved to have high plasticity, together with a constant proliferative rate and a remarkable ability to differentiate towards different cell lines.

\section{Conclusion}

This study contributes to the characterization of AD-MSCs derived from rat and to the understanding of their nature. Since the rat represents an ideal model to understand tissue reparative mechanisms and it is also useful for translational medicine studies, results obtained in this study emphasize once again how
AD-MSCs derived from rat represent an ideal tool to better understand the mechanisms at the basis of cell therapy and for the development of regenerative medicine approaches.

\section{References}

[1]. Caplan AI, Bruder SP (2001) Mesenchymal stem cells: building blocks for molecular medicine in the 21st century. Trends Mol Med 7(6): 259-264.

[2]. Guercio A, Di Marco P, Casella S, Cannella V, Russotto L, et al. (2012) Production of canine mesenchymal stem cells from adipose tissue and their application in dogs with chronic osteoarthritis of the humeroradial joints. Cell Biol Int 36(2): 189-194.

[3]. Leo AJ, Grande DA (2006) Mesenchymal stem cells in tissue engineering. Cells Tissues Organs 183(3): 112-122.

[4]. Bottai D, Fiocco R, Gelain F, Defilippis L, Galli R, et al. (2003) Neural stem cells in the adult nervous system. J Hematother Stem Cell Res 12(6): 655-670.

[5]. Guercio A, Di Bella S, Casella S, Di Marco P, Russo C, et al. (2013) Canine mesenchymal stem cells (MSCs): characterization in relation to donor age 
and adipose tissue-harvesting site. Cell Biol Int 37(8): 789-798.

[6]. Koch TG, Heerkens T, Thomsen PD, Betts DH (2007) Isolation of mesenchymal stem cells from equine umbilical cord blood. BMC Biotechnol 7: 26.

[7]. Wickham MQ, Erickson GR, Gimble JM, Vail TP, Guilak F (2003) Multipotent stromal cells derived from the infrapatellar fat pad of the knee. Clin Orthop Relat Res (412): 196-212.

[8]. Williams JT, Southerland SS, Souza J, Calcutt AF, Cartledge RG (1999) Cells isolated from adult human skeletal muscle capable of differentiating into multiple mesodermal phenotypes. Am Surg 65(1): 22-26.

[9]. Parker AM, Katz AJ (2006) Adipose-derived stem cells for the regeneration of damaged tissues. Expert Opin Biol Ther 6(6): 567-578.

[10]. Black LL, Gaynor J, Gahring D, Adams C, Aron D, et al. (2007) Effect of adipose-derived mesenchymal stem and regenerative cells on lameness in dogs with chronic osteoarthritis of the coxofemoral joints: a randomized, double-blinded, multicenter, controlled trial. Vet Ther 8(4): 272-284.

[11]. Tonchev AB, Bełtowski J, Fiore M, Rančić G, Chechi K, et al. (2010) Adipobiology of stem cell-based therapy: secretome insight. Biomed Rev 21: 57-63.

[12]. Schaffler A, Buchler C (2007) Concise review: adipose tissue-derived stromal cells--basic and clinical implications for novel cell-based therapies. Stem Cells 25(4): 818-827.

[13]. Fraser JK, Wulur I, Alfonso Z, Hedrick MH (2006) Fat tissue: an underappreciated source of stem cells for biotechnology. Trends Biotechnol 24(4): $150-154$.

[14]. Nakagami H, Morishita R, Maeda K, Kikuchi Y, Ogihara T, et al. (2006) Adipose tissue-derived stromal cells as a novel option for regenerative cell therapy. J Atheroscler Thromb 13(2): 77-81.

[15]. Baglioni S, Francalanci M, Squecco R, Lombardi A, Cantini G, et al. (2009) Characterization of human adult stem-cell populations isolated from visceral and subcutaneous adipose tissue. FASEB J 23(10): 3494-3505.

[16]. Colleoni S, Bottani E, Tessaro I, Mari G, Merlo B, et al. (2009) Isolation, growth and differentiation of equine mesenchymal stem cells: effect of donor, source, amount of tissue and supplementation with basic fibroblast growth factor. Vet Res Commun 33(8): 811-821.

[17]. Coli A, Nocchi F, Lamanna R, Iorio M, Lapi S, et al. (2011) Isolation and characterization of equine amnion mesenchymal stem cells. Cell Biol Int Rep 18(1): 23-29.

[18]. Neupane M, Chang CC, Kiupel M, Yuzbasiyan-Gurkan V (2008) Isolation and characterization of canine adipose-derived mesenchymal stem cells. Tissue Eng 14(6): 1007-1015.

[19]. Vieira NM, Brandalise V, Zucconi E, Secco M, Strauss BE, et al. (2010) Isolation, characterization, and differentiation potential of canine adiposederived stem cells. Cell Transplant 19(3): 279-289.

[20]. Taha MF, Hedayati V (2010) Isolation, identification and multipotential differentiation of mouse adipose tissue-derived stem cells. Tissue Cell 42(4): 211-216.

[21]. Polisetti N, Chaitanya VG, Babu PP, Vemuganti GK (2010) Isolation, characterization and differentiation potential of rat bone marrow stromal cells. Neurol India 58(2): 201-208.

[22]. Altomare R, Cannella V, Abruzzo A, Palumbo VD, Damiano G, et al. (2014) Obtaining mesenchymal stem cells from adipose tissue of murin origin: experimental study. Int J Stem Cell Res Transplant 2(5): 1-5.

[23]. Livak KJ, Schmittgen TD (2001) Analysis of relative gene expression data using Real-Time quantitative PCR and the $2^{-\triangle \Delta C T}$ Method. Methods 25(4): 402-408.

[24]. Case J, Horvath TL, Ballas CB, March KL, Srour EF (2008) In vitro clonal analysis of murine pluripotent stem cells isolated from skeletal muscle and adipose stromal cells. Exp Hematol 36(2): 224-234.

[25]. Izadpanah R, Joswig T, Tsien F, Dufour J, Kirijan JC, et al. (2005) Characterization of multipotent mesenchymal stem cells from the bone marrow of rhesus macaques. Stem Cells Dev 14(4): 440-451. 